\title{
Método Aplicado vs. Direto: dimensionamento de um sistema de distribuição de ar para instalações HVAC
}

Applied vs. Straight method: air distribution duct design for HVAC installations.

\author{
B. E. A. Pereira ${ }^{1}$; M. M. Rebelato ${ }^{1}$; G. S. Velasques ${ }^{1}$; A. F. Porte ${ }^{2}$; \\ M. M. Galarça $a^{2,3 *}$ \\ ${ }^{I}$ Tecnologia em Refrigeração e Climatização, IFRS-Campus Rio Grande, CEP: 96201-460, Rio Grande-RS, Brasil. \\ ${ }^{2}$ Engenharia Mecânica/LATESC/IFRS-Campus Rio Grande, CEP:96201-460, Rio Grande-RS, Brasil \\ ${ }^{3}$ Programa de Pós Graduação em Modelagem Computacional/FURG, CEP:96201-900, Rio Grande-RS, Brasil
}

*marcelo.galarca@ riogrande.ifrs.edu.br

(Recebido em 07 de novembro de 2016; aceito em 23 de maio de 2017)

\begin{abstract}
O presente trabalho aborda o processo de dimensionamento de dutos para distribuição de ar em sistemas de ventilação e/ou climatização. São comparadas duas formas de se efetuar o dimensionamento: na primeira, pela utilização de dados em tabelas e gráficos construídos a partir de experimentos empíricos; em outra, a partir da solução das equações fundamentadas na mecânica dos fluidos e correlações empíricas. Neste trabalho as abordagens em relação aos dois procedimentos de cálculo para dimensionamento são definidas como Método Aplicado e Método Direto, respectivamente. As duas formas de solução são aplicadas aos três principais métodos de dimensionamento de sistema de dutos: velocidade arbitrária, perda de carga constante e recuperação da pressão estática. O dimensionamento é feito para um sistema de dutos hipotético. São avaliados o comportamento da perda de carga para cada um dos métodos de dimensionamento e procedimento de solução, bem como as dimensões finais do sistema de dutos. Os resultados mostram desvios percentuais pouco significativos, em termos absolutos, em relação aos procedimentos de solução adotados.

Palavras-chave: dimensionamento de dutos, métodos de dimensionamento, métodos aplicado e direto.
\end{abstract}

This paper addresses the duct design process for distribution of air ventilation and / or air conditioning. It is made a comparison between two ways of designing: the first one, by using data from tables and graphs constructed based on empirical experiments; another one, from the solution of fluid mechanics equations. In this work, the approaches to both calculation procedures for sizing are defined as Applied and Straight Methods, respectively. The two types of solution are applied to the three main ducts system design methods: arbitrary velocity, constant head loss and recovery of static pressure. The design is runed for a hypothetical duct system. The results consider the head loss behavior for each design methods and settlement solution and the final dimensions of the duct system. The results show no significant deviations, about absolute values, in relation to the settlement solution adopted.

Keywords: pipeline design, sizing methods, Applied and straight methods

\section{INTRODUÇÃO}

Com a escassez crescente de energia em conjunto com a crescente preocupação em relação ao meio ambiente, cada vez mais se buscam sistemas de aquecimento, ventilação e ar-condicionado (HVAC, Heating, Ventilation and Air Conditioning) os quais possam operar com limites de aumento na eficiência energética sem que se perca a qualidade do ar no interior de uma edificação. O primeiro passo na obtenção de um sistema que faça um melhor aproveitamento da energia, no objetivo de distribuir o ar na edificação, diz respeito à forma como os dutos são dimensionados e balanceados. Conforme Sugarman (2006) [1], um balanceamento preciso melhora significativamente a eficiência do sistema pela minimização de desvios na distribuição de ar para cada zona, uma vez que o consumo de energia para o tratamento do ar é cubicamente proporcional às taxas de vazão.

Durante o processo de dimensionamento, o balanceamento é parcialmente considerado de forma implícita nos métodos de dimensionamento [2]. Desta forma, quanto menos aproximações puderem 
ser consideradas nos cálculos de dimensionamento de sistemas HVAC, maiores as chances de se evitar a necessidade de um balanceamento trabalhoso e, por consequência, oneroso. Segundo Enteria e Mizutani (2011) [3], cerca de 50\% da demanda de energia em edificações comerciais é utilizada para fornecer condições de conforto térmico. Ainda, como a maioria das pessoas gastam mais do que $90 \%$ de seu tempo no interior das edificações, o desenvolvimento de sistemas HVAC eficazes é ponto significativo no objetivo de melhor aproveitamento energético dos equipamentos [4]. Um bom dimensionamento e boa configuração de um sistema deve se basear na carga de refrigeração (para sistemas de ar-condicionado), vazão de renovação de ar (para sistemas de ventilação), além da queda de pressão que deverá ser vencida [5].

Tem sido amplamente reconhecido que a fim de permitir o conceito de eficiência energética em equipamentos de HVAC é fundamental desenvolver modelos precisos para testes e estratégias de controle do consumo relativo de energia [6,7].

Como em qualquer sistema de engenharia (processo de desenvolvimento de sistemas de distribuição de ar para conforto térmico), existem as incertezas inerentes aos cálculos e considerações de projeto. Incertezas podem levar um sistema a ser super ou subdimensionado. Um projeto superdimensionado aumentará o custo inicial e operacional do sistema. O projeto sendo subdimensionado irá, sem dúvida, sacrificar o conforto térmico da edificação nos dias em que o sistema HVAC não fornecer capacidade de resfriamento ou aquecimento. Assim, é necessário, em maior ou menor grau aceitar que as incertezas afetam os sistemas de distribuição de ar [8].

Existem diversas literaturas as quais podem ser consultadas para orientar em um bom dimensionamento [9, 10, 11 e 12]. Os métodos de dimensionamento de tais sistemas mais comumente utilizados são: Velocidade Arbitrária, Perda de Carga Constante e Recuperação da Pressão Estática. Existem recomendações para a utilização deste ou aquele, no entanto para todos eles existem duas formas de se efetuar o dimensionamento: pela utilização de gráficos e tabelas, com dados obtidos através de fabricantes de equipamentos e/ou por meios empíricos (procedimento empírico); ou pela solução das equações fundamentais da mecânica dos fluidos, buscando resolver de forma direta o problema do dimensionamento a partir de uma queda de pressão a ser vencida e uma conhecida vazão de operação (procedimento clássico) [13].

O dimensionamento através da metodologia aplicada é relativamente simples, porém pode carregar muita incerteza, uma vez que depende diretamente da leitura do projetista nos gráficos e tabelas. A leitura de alguns parâmetros, que deve ser feita diretamente em gráficos pode ficar comprometida devido às aproximações inerentes aos dados, bem como pela imprecisão da, literalmente, visão de quem lê e interpreta os gráficos.

A utilização dos conjuntos de equações referentes ao estudo das perdas por atrito, para escoamentos, da mecânica dos fluidos exige alguns procedimentos algébricos de forma a resolver as equações conforme a característica de cada método de dimensionamento. Uma vez que o equacionamento esteja algebricamente organizado, a solução do sistema de equações pode ser feita pela utilização de um computador, pois envolve alguns processos iterativos.

Este trabalho tem por objetivo avaliar os resultados de dimensionamento, de um sistema de distribuição de ar hipotético, para as três formas de dimensionamento mais conhecidas através de duas metodologias de solução distintas: aplicada e direta. A partir dos resultados verificam-se as diferenças entre as formas de solução e se estas podem se apresentar significativamente diferentes em termos de projeto.

\section{PERDA DE CARGA EM SISTEMAS DE DUTOS}

Para um sistema HVAC, os dutos de transporte do ar oferecem certa resistência ao escoamento. Essa resistência é comumente chamada de perda de carga. Fisicamente significa a perda de energia mecânica pela unidade de massa de um fluido causada pelo atrito das paredes internas e da turbulência do escoamento entre dois pontos de um duto. A perda de carga nada mais é do que uma variação de pressão entre pontos de uma tubulação [14 e 15].

Desta forma, um sistema que venha a oferecer uma menor perda de carga, para a mesma vazão de instalação requerida, irá operar com um custo energético menor. 
A perda de carga total, $h_{1 t}$, de um sistema de dutos é estimada como a soma das perdas principais, $h_{1}$, mais as perdas secundárias, $h_{m}$, Eq.(1). As perdas principais constituem a maior parte da perda de carga total em relação às secundárias, pois normalmente os comprimentos das tubulações possuem grandes extensões. As unidades da perda de carga são comumente apresentadas em termos de pressão ou coluna de fluido (Pa ou m). Neste trabalho é convencionada a unidade em Pascal.

$$
h_{l t}=h_{l}+h_{l m}
$$

A perda de carga principal, $h_{l}$, é provocada pelo efeito do atrito da parede do duto com o escoamento do fluido plenamente desenvolvido entre dois pontos, em um tubo de seção constante. Esta depende diretamente do regime de escoamento - laminar ou turbulento. Para o estudo neste trabalho é adotado o regime turbulento, por ser mais comum em aplicações reais em sistemas de distribuição de ar, no qual a Eq.(2) é aplicada para a determinação da perda de carga sob estas condições.

$$
h_{l}=f \frac{L}{D} \frac{\rho u^{2}}{2}
$$

onde $L$ é comprimento do trecho reto em metros, $D$ é o diâmetro do trecho para tubos circulares ou $D_{h}$, diâmetro hidráulico, para tubos retangulares em metros, $f$ é o fator de atrito adimensional, $u$ é a velocidade média do escoamento em $\mathrm{m} / \mathrm{s}$ e $\rho$ é massa específica do fluido $\mathrm{em} \mathrm{kg} / \mathrm{m}^{3}$.

As perdas de carga secundárias, $h_{l m}$, são causadas devido ao atrito do fluido em escoamento no interior de acessórios, tais como difusores, curvas, válvulas, etc. O cálculo desta perda pode ser efetuado pelo conhecimento de um comprimento equivalente em metro de tubulação reta, $L_{e q}$, dos acessórios. Estes valores são, comumente, fornecidos através de tabelas ou gráficos construídos a partir de dados experimentais (seja pelos próprios fabricantes de acessórios, ou dados generalistas disponíveis em literatura, de acordo com o acessório). Uma vez conhecendo o comprimento equivalente, a equação para determinação da perda de carga secundária, Eq.(3), torna-se uma variação muito próxima à Eq.(2).

$$
h_{l m}=f \frac{L_{e q}}{D} \frac{\rho u^{2}}{2}
$$

Desta forma, é explícita a necessidade de se conhecer o fator de atrito, $f$, na busca pela perda de carga de qualquer sistema fluidodinâmico para escoamento em regime turbulento. Já é de conhecimento amplo que $f$ possui dependência direta do tipo de material do qual o duto é feito, pois disto depende a rugosidade superficial. Outo ponto já bem estabelecido é que esta constante apresenta dependência, igualmente, do número de Reynolds. Valores de rugosidades são dados fornecidos por fabricantes, usualmente disponíveis na literatura correlata. Uma aproximação comum é considerar um valor significativamente baixo para $f$, e tratar o duto como sendo liso [14 e 16]. Assim temos o fator de atrito como uma função dependente destas variáveis, Eq.(4).

$$
f=\phi(\operatorname{Re}, \varepsilon / D)
$$

onde $\phi$ representa uma função qualquer, Re é o número de Reynolds e a razão entre a rugosidade, $\varepsilon$ e o diâmetro, $D$ é chamada de rugosidade relativa. 
Desta forma, fica evidenciado que o valor de $f$ é totalmente dependente de dados empíricos. A forma de simplificar os cálculos relativos às perdas de carga em um sistema se dá a partir da utilização de correlações, gráficos e/ou tabelas, ambos gerados a partir de experimentos. Assim, é importante estar ciente de que esta variável já traz consigo alguma incerteza incorporada. No entanto, a ampla literatura apresenta muitas opções para o cálculo da mesma. Dentre os trabalhos mais conhecidos encontram-se as proposições de Churchill (1973) [17], Churchill (1977) [18], Moody (1944) [19], Moody (1947) [20], Colebrook (1939) [21] e Wood (1966) [22].

Para este trabalho são utilizadas as correlações de Wood (1966) [22], Eq.(5); e Colebrook (1939) [21], Eq.(6). A Eq.(5) é aplicável em uma faixa de números de Reynolds e de rugosidade relativa de $4 \times 10^{3}-5 \times 10^{7}$ e $1 \times 10^{-5}-4 \times 10^{-2}$, respectivamente.

$$
f=a+b(\mathrm{Re})^{-c}
$$

onde,

$$
\begin{aligned}
& a=0,53\left(\frac{\varepsilon}{D}\right)+0,094\left(\frac{\varepsilon}{D}\right)^{0,225} \\
& b=88\left(\frac{\varepsilon}{D}\right)^{0,44} \\
& c=1,62\left(\frac{\varepsilon}{D}\right)^{0,1344}
\end{aligned}
$$

Uma correlação mais apropriada, que permite que os cálculos sejam realizados com auxílio de computador, é a proposta por Colebrook (1939) [21], Eq.(6), a qual exige processo iterativo.

$$
\frac{1}{\sqrt{f}}=-2,0 \log \left(\frac{\varepsilon / D}{3,7}+\frac{2,51}{\operatorname{Re} \sqrt{f_{0}}}\right)
$$

onde $f_{0}$ é uma estimativa inicial para a solução iterativa, igualmente proposta por Colebrook (1939)[21], dada pela Eq.(7), já que se trata de um problema com solução iterativa.

$$
f_{0}=0,25\left[\log \left(\frac{\varepsilon / D}{3,7}+\frac{5,7}{\mathrm{Re}^{0,9}}\right)\right]^{-2}
$$

\section{METODOLOGIA DE DIMENSIONAMENTO}

Conforme já mencionado anteriormente, o presente trabalho traz os dimensionamentos obtidos pelos três principais métodos disponíveis na literatura, utilizando-se para isto duas formas de solução distintas: uma pela aplicação de gráficos, tabelas e correlações; outra pela solução das equações fundamentais da teoria de Bernoulli em conjunto às correlações empíricas. As duas formas de solução do dimensionamento são melhores descritas na sequência deste trabalho. A seguir, os métodos de dimensionamento, aqui, abordados são:

(a) Método da Velocidade Arbitrária (ou Velocidade Constante): Consiste em adotar as velocidades recomendadas, não prevendo o equilíbrio das pressões dinâmicas nos difusores de ar. 
(b) Método da Perda de Carga Constante: Este método tem o intuito em calcular os dutos de forma a proporcionar que tenham a mesma perda de carga por unidade de comprimento ao longo do sistema.

(c) Método da recuperação da pressão estática: Este método dimensiona o duto afim de que o aumento da pressão estática em cada trecho ou difusor de ar compense as perdas por atrito na seção seguinte do duto. Devido a isto, a pressão estática será a mesma para cada difusor e no começo de cada trecho.

O sistema proposto para este estudo, ilustrado na Figura (1), é constituído de um segmento de dutos. No primeiro trecho o ventilador insufla o ar a uma vazão constante de $9000 \mathrm{~m}^{3} / \mathrm{h}$ pelo duto principal, que divide essa a vazão total para três ramificações, distribuindo $3000 \mathrm{~m}^{3} / \mathrm{h}$ de ar para cada ramificação. Cada ramificação tem seis bocas de insuflamento de $500 \mathrm{~m} / \mathrm{h}$ cada.

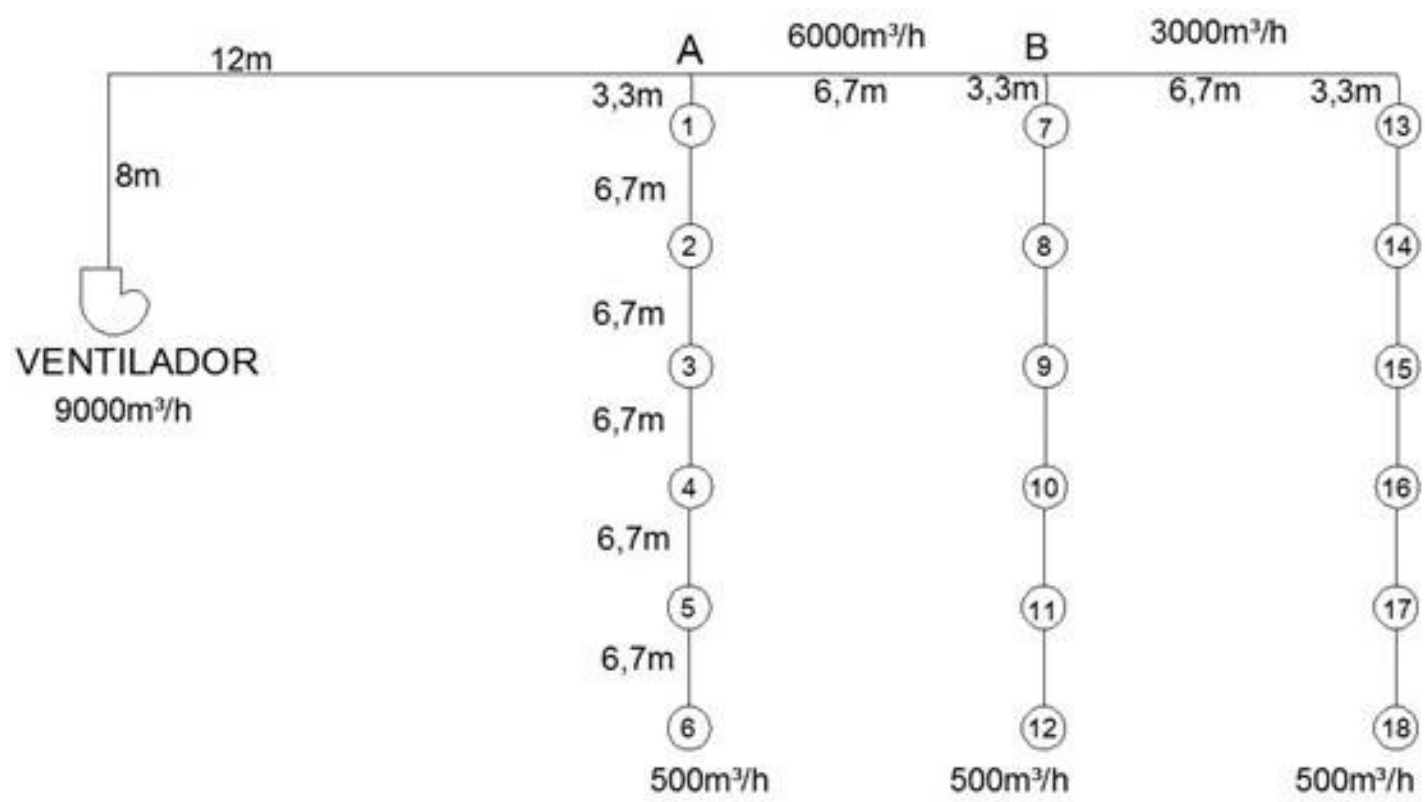

Figura 1. Sistema de distribuição de ar (Adaptado de Carrier,1972) [13]

Determina-se uma velocidade inicial no duto principal, supondo o sistema de uma instalação comercial de baixa pressão, negligenciando o sistema de retorno, a velocidade adotada é de $9 \mathrm{~m} / \mathrm{s}$ [22]. A temperatura de insuflamento, para fins de propriedades físicas do ar, é de $15^{\circ} \mathrm{C}$. Nesta condição a massa específica do ar é de $1,2 \mathrm{~kg} / \mathrm{m}^{3}$, a viscosidade dinâmica de $1,85 \times 10^{-5}$ Pa.s e as paredes internas do sistema de dutos são consideradas lisas, com rugosidade da ordem de $1 \times 10^{-5}$ $\mathrm{m}$. É importante deixar claro, neste ponto, que por se tratar de uma análise restrita às formas de cálculo para dimensionamento de dutos, não são considerados os ganhos térmicos, fugas de ar e sistema de retorno.

\subsection{PROCEDIMENTOS DE CÁlCULO}

O dimensionamento de sistemas de dutos, embora existam os três métodos já bastante difundidos pela literatura, ainda gera muita dúvida em relação a quais os procedimentos seguir para um bom projeto. Profissionais com muitos anos de prática geralmente tem suas próprias metodologias a partir daquelas já existentes. Com a crescente utilização do computador na solução de problemas em engenharia, atualmente é possível encontrar alguns softwares voltados para o projeto de sistemas de distribuição de ar e climatização. No entanto, mesmo em relação aos programas computacionais é possível observar as diferentes maneiras de apresentar a mesma solução para um dimensionamento final de um sistema de dutos. Independentemente destas variáveis, o que se pode identificar claramente são dois caminhos distintos: (1) fazer uso de documentos gráficos, tais como tabelas e figuras, os quais relacionam graficamente propriedades como velocidade no interior do duto, comprimento de trecho, tipos de acessórios, etc., com as 
dimensões e variações de pressão num sistema de duto. Esta forma é comumente utilizada, principalmente quando se deseja ter uma estimativa inicial a respeito do quão potente deverá ser este ou aquele equipamento num sistema HVAC. Por ser um procedimento no qual poucos cálculos efetivamente são feitos (já que está tudo em gráficos e/ou tabelas), o mesmo se torna amplamente difundido; (2) utilizar-se do equacionamento para determinação da queda de pressão, a partir das relações de Bernoulli para velocidade e pressão. Manipulando algebricamente as equações fundamentais, e aplicando as mesmas em um sistema de escoamento no interior de dutos. Por se tratar de escoamento turbulento, não será possível deixar de usar correlações empíricas para resolver o comportamento do fator de atrito, $f$. Bem como, contabilizar a influência de curvas (e/ou acessórios) é, igualmente, feito com base em relações matemáticas algébricas. Porém, desta forma, uma vez fazendo uso de correlações confiáveis e de alguma habilidade em termos de programação para engenharia, a solução para o dimensionamento de um sistema de dutos pode ser feito, de forma completa, sem necessitar da utilização de qualquer elemento gráfico pelo qual se incorreria no acréscimo de erro devido a imprecisão da visão e interpretação humana.

Uma vez esclarecidas as duas distintas formas na solução de um mesmo problema. Assume-se, deste ponto em diante, como Método Aplicado e Método Direto, respectivamente, conforme descrito no parágrafo anterior. O Método Direto foi implementado em linguagem de programação Phyton. O trabalho se propõe a comparar, para um sistema hipotético, os resultados em termos de dimensões finais e perda de carga teórica em relação a ambas as formas de solução.

\subsubsection{VELOCIDADE ARBITRÁRIA (MÉTODO APLICADO)}

Em relação às três formas de dimensionamento, esta é certamente a mais simples. No entanto, é recomendada para sistemas pequenos, geralmente sem ramificações, ou contendo, no máximo três ramificações com poucas saídas. O método, em sua forma de solução, se baseia basicamente na conservação de massa. Desta forma as quedas de pressão ao longo do sistema são consequência direta do atrito gerado pelo escoamento nas paredes dos dutos.

No dimensionamento a partir deste método, inicialmente determinam-se as vazões (usualmente com base na carga térmica do ambiente e/ou do volume necessário para renovação do ar) para cada trecho do sistema. Uma vez que a velocidade é arbitrária, a mesma é um valor conhecido e comumente adotado a partir de recomendações de norma [11]. Desta forma, a partir da equação da continuidade, Eq.(8), obtém-se a área da seção transversal do duto, $A$, em m².

$$
Q=\int_{A} V d A=u A
$$

onde $Q$ é a vazão volumétrica em $\mathrm{m}^{3} / \mathrm{s}, V$ é uma função da velocidade (ou campo de velocidades) e $u$ a velocidade média do escoamento em $\mathrm{m} / \mathrm{s}$. Uma vez que as equações para determinação das perdas de carga a seção transversal necessitam do diâmetro, $D$, faz-se necessário adotar uma relação tal como o diâmetro hidráulico, $D_{h}$, para seção retangular do duto, ou alguma outra relação que faça essa aproximação. Uma correlação útil para o diâmetro hidráulico é apresentada pela Eq.(9). Para dutos circulares o diâmetro é obtido da relação direta com a área.

$$
D_{h}=\frac{(H L)^{0,625}}{(H+L)^{0,25}}
$$

Para escoamento turbulento (adotado neste trabalho) o fator de atrito é determinado pela aplicação da Eq.(5). O mesmo ainda pode ser obtido através de diagramas gráficos, como o bem conhecido Diagrama de Moody [19]. Neste último caso, além do erro intrínseco às correlações as quais geraram o diagrama, acrescenta-se o erro visual humano. A perda de carga (ou queda de pressão) total do sistema é obtida pelas Eqs.(1), (2) e (3), que nada mais é do que o somatório de todas as perdas obtidas para cada trecho do sistema. Neste trabalho os valores de comprimentos equivalentes são obtidos conforme tabelas fornecidas por Carrier (1972) [13]. Para a primeira 
(próxima ao ventilador) e segunda (na última ramificação) curvas do sistema de distribuição, Figura (1), os valores são de 3,42 e 2,05 metros, respectivamente.

\subsubsection{PERDA DE CARGA CONSTANTE (MÉTODO APLICADO)}

Para o próximo método, a seção do primeiro trecho é a mesma que aquela calculada anteriormente, através do conceito da conservação de massa, Eq.(8). As dimensões subsequentes são obtidas pela utilização de correlações, gráficos ou tabelas. Uma literatura comumente utilizada é Carrier (1972) [13]. Nesta, as dimensões de dutos são obtidas a partir de uma tabela, a qual faz relação direta entre área e vazão volumétrica para cada trecho e sua respectiva área de seção do duto, em função do percentual nas diferenças entre o trecho conhecido e o próximo (seção de duto).

A queda de pressão é obtida através de um diagrama de perda de carga por metro de trecho reto $\left(\mathrm{Pa} / \mathrm{m}\right.$ ou $\left.\mathrm{mmH}_{2} \mathrm{O} / \mathrm{m}\right)$, o qual é amplamente utilizado na literatura [22]. Uma vez obtida a perda de carga para cada metro de tubulação, a perda total é dada pelo produto entre o valor unitário e o comprimento mais longo percorrido pelo ar (do ventilador até a última saída de ar, difusor 18, Figura (1)). Este procedimento é amplamente utilizado por diversas literaturas [12, 15 e 22]. Ainda, para composição da perda de carga total são somados os comprimentos equivalentes das curvas do segmento de maior comprimento, pela aplicação da Eq.(3). A velocidade média, $u$, é resultado direto da vazão volumétrica, a qual será conhecida a partir das tabelas aplicadas ao método de dimensionamento [13], conforme mencionado anteriormente.

Neste trabalho o valor estimado para a perda de carga unitária, a partir do diagrama [22] é de $0,14 \mathrm{mmH}_{2} \mathrm{O} / \mathrm{m}$.

Além da metodologia apresentada por Carrier (1972) [13], uma alternativa que faz uso apenas de correlações diretas entre vazão e dimensões do duto é apresentada pela ASHRAE (2013) [11]. É um procedimento baseado em dados empíricos e com correlações de aplicação direta. Estas são divididas em dois grupos: sistemas de alta pressão e sistemas de baixa pressão. Neste trabalho, o dimensionamento considera o segundo grupo, onde a velocidade máxima é de $12 \mathrm{~m} / \mathrm{s}$ [11].

Para o sistema de baixa pressão o cálculo do diâmetro é dado pela Eq.(10) onde a vazão (Q) é definida em $\mathrm{L} / \mathrm{s}$, e o diâmetro, $D$, é dado em metros.

$$
D=\frac{32\left(Q^{0,38}\right)}{1000}
$$

A perda de carga total é calculada da mesma forma anteriormente exposta.

\subsubsection{RECUPERAÇÃO DA PRESSÃo ESTÁTICA (MÉTODO APLICADO)}

O cálculo da perda de carga neste método também faz uso do procedimento para obtenção da perda de carga unitária, como no método anterior, onde se obteve o valor de $0,14 \mathrm{mmH}_{2} \mathrm{O} / \mathrm{m}$. Porém, nesse caso, a perda de carga por metro, é multiplicada apenas pelo somatório do comprimento da seção reta e dos comprimentos equivalentes de curva(s) e/ou acessórios do primeiro trecho, ou seja, do ventilador até o ponto da primeira ramificação. Conforme procedimento reconhecido em literatura [13 e 15].

A área do primeiro trecho é calculada pela relação direta de área e vazão dada pela equação da conservação de massa, Eq.(8). A determinação das áreas dos demais trechos, a fim de manter uma recuperação de pressão estática, é feita pela obtenção das velocidades a partir do diagrama da relação $L / Q$, o qual correlaciona a vazão atual do trecho com o comprimento equivalente deste, e o gráfico da Recuperação Estática em Baixa Velocidade, o qual relaciona a velocidade atual com a relação $L / Q$ e a velocidade do trecho anterior. Este procedimento é detalhado em Costa (2005) [12], Carrier (1972) [13] e (Miller e Miller, 2014) [15]. O importante aqui é ressaltar que praticamente todos os dados de cada etapa do dimensionamento são obtidos pela utilização de gráficos e que, desta forma, a leitura correta e precisa destes tem influência direta do olho humano. Fica evidente a necessidade de manuseio de quantidade significante de material gráfico (impresso ou não) para se efetuar o dimensionamento de um sistema de dutos por tal metodologia. 


\subsubsection{VELOCIDADE ARBITRÁRIA (MÉTODO DIRETO)}

Para o dimensionamento a partir do Método Direto em relação a Velocidade Arbitrária a diferença se dá apenas nas formas de obtenção do fator de atrito, $f$, o qual se dá pela aplicação das Eqs.(6) e (7), bem como na maneira de se levar em consideração a existência de acessórios (curvas, tês, etc.) e suas respectivas perdas de carga. Pelo Método Direto, não se faz uso de tabelas ou gráficos, mas de correlações empíricas utilizadas em conjunto com Bernoulli e as equações de balanço. Desta forma a Eq.(1) dá lugar a aplicação da Eq.(11)[11 e 15].

$$
h_{l t}=\rho\left(f \frac{L}{D}+0,1 \frac{\theta}{90}\right) \frac{u^{2}}{2}
$$

onde $\theta$, única informação nova, é o ângulo total de curvatura (todas as curvas) no trecho. Em sistemas que fazem uso de outros acessórios, tais como defletores, pás guias, filtros, etc., a perda de carga deve ser somada aos resultados obtidos pela Eq.(11). Para o caso estudado neste trabalho, apenas curvas estão sendo consideradas.

\subsubsection{PERDA DE CARGA CONSTANTE (MÉTODO DIRETO)}

O dimensionamento pela Perda de Carga Constante, a partir do Método Direto necessita de uma manipulação algébrica de forma a resolverem-se as Eqs.(2) e (3). Uma vez que para quaisquer dos métodos de dimensionamento, o primeiro trecho sempre possui a velocidade (consequentemente vazão) e seção transversal conhecidas, é necessário que se dimensione o trecho posterior, a partir das condições do anterior. Assim, para se garantir perda de carga constante, a igualdade na queda de pressão por metro do trecho anterior e do posterior deve ser mantida. Logo, pela Eq.(2) (e de forma análoga, para a Eq.(3)), tem-se:

$$
f \rho \frac{L}{D_{1-2}} u_{1-2}^{2}=f \rho \frac{L}{D_{2-3}} u_{2-3}^{2}=\Delta p_{1-2}=\Delta p_{2-3}
$$

onde os subíndices 1-2 e 2-3 representam trechos de seções de dutos anterior e posterior, respectivamente, e $L$ neste caso é uma unidade de comprimento. Para casos em que se considere a existência de curvas presentes nos trechos, a contabilização destas se dá pela inserção das parcelas referentes aos ângulos de curvaturas. Desta forma, a Eq.(12) é reescrita como:

$$
f \rho\left(\frac{L}{D_{1-2}}+0,1 \frac{\theta}{90}\right) u_{1-2}^{2}=f \rho\left(\frac{L}{D_{2-3}}+0,1 \frac{\theta}{90}\right) u_{2-3}^{2}
$$

Uma vez que o fator de atrito, $f$, é obtido de forma iterativa, a solução da Eq.(13) para cada trecho do sistema é, igualmente, iterativa, pois depende da solução de $f$, e este, depende da velocidade média tanto no trecho anterior quanto posterior (não conhecida).

\subsubsection{RECUPERAÇÃO DA PRESSÃO ESTÁTICA (MÉTODO DIRETO)}

O método propõe a recuperação da pressão estática com a redução da velocidade no sentido de escoamento, tendo por objetivo manter constante o nível estático entre os trechos. A perda de carga total do sistema é obtida pelo produto entre a perda de carga por metro e o comprimento do primeiro trecho (ventilador até o ponto A). Na equação da perda de carga é adicionado uma variável chamada de "constante de recuperação", $R$, a qual pode variar de 75 a 100\% [11 e15]. Embora não seja possível fisicamente recuperar toda pressão estática de um trecho para outro, no presente trabalho, por simplicidade, adota-se recuperação total, ou seja, $R=1$. Da mesma forma como no método 
anterior, o rearranjo das equações gera um problema de fechamento o qual só pode ser resolvido iterativamente. A equação de perda de carga, considerando curvas e a recuperação da pressão estática é dada, então, por:

$$
\begin{aligned}
& R\left(\frac{\rho u_{1-2}^{2}}{2}-\frac{\rho u_{2-3}^{2}}{2}\right)=f \frac{L_{2-3}}{D_{2-3}} \frac{\rho u_{2-3}^{2}}{2}+0,1 \frac{\theta}{90} \frac{\rho u_{2-3}^{2}}{2} \\
& R u_{1-2}^{2}=\left(f \frac{L_{2-3}}{D_{2-3}}+0,1 \frac{\theta}{90}+R\right) u_{2-3}^{2}
\end{aligned}
$$

\section{RESULTADOS E DISCUSSÃO}

Os resultados obtidos pelos procedimentos de cálculo apresentados anteriormente, com os três métodos para dimensionamento, são analisados e comparados entre si em função das perdas de carga e dimensões dos trechos do sistema de dutos.

Primeiramente são observadas as áreas para todos os trechos do sistema em relação a cada um dos três métodos de dimensionamento, através do Método Aplicado, Figura (2), e Método Direto, Figura (3). O comportamento apresentado em ambos os gráficos é esperado condizente com o fenômeno físico. As diferenças maiores se dão em relação aos métodos da velocidade arbitrária e da recuperação da pressão estática, independentemente da metodologia de cálculo adotada. É observado, na Figura (3), que os trechos finais referentes a última ramificação (difusor 13 até 18), na metodologia de solução direta os dimensionamentos por perda de carga constante e recuperação da pressão estática apresentaram tendências e valores muito próximos. Uma possível explicação para isto se dá pelo fato de que como os trechos entre cada difusor na ramificação são curtos e de mesmo comprimento, o escoamento, uma vez que a perda de carga é pequena, recupera em certo grau a pressão estática do trecho anterior pela redução da área das seções subsequentes. Este fato torna o dimensionamento final muito próximo para ambos os métodos. O mesmo comportamento não é observado quando se utilizou o procedimento baseado em ábacos e tabelas, ou Método Aplicado.

Outra observação evidente é que as diferenças na seção transversal do trecho "AB" entre os três métodos são mais acentuadas quando o Método Direto é adotado, principalmente em relação ao dimensionamento por recuperação da pressão estática. Este comportamento pode ser gerado pelo fato de que este método (recuperação estática), quando faz uso maior de gráficos e tabelas para efetuar o dimensionamento, pode aumentar as discrepâncias em relação a uma metodologia que siga uma forma de solução mais direta em relação a aplicação das equações de balanço e a teoria de Bernoulli. No decorrer dos procedimentos de cálculo, nos trechos seguintes, a convergência para valores semelhantes ocorre. 


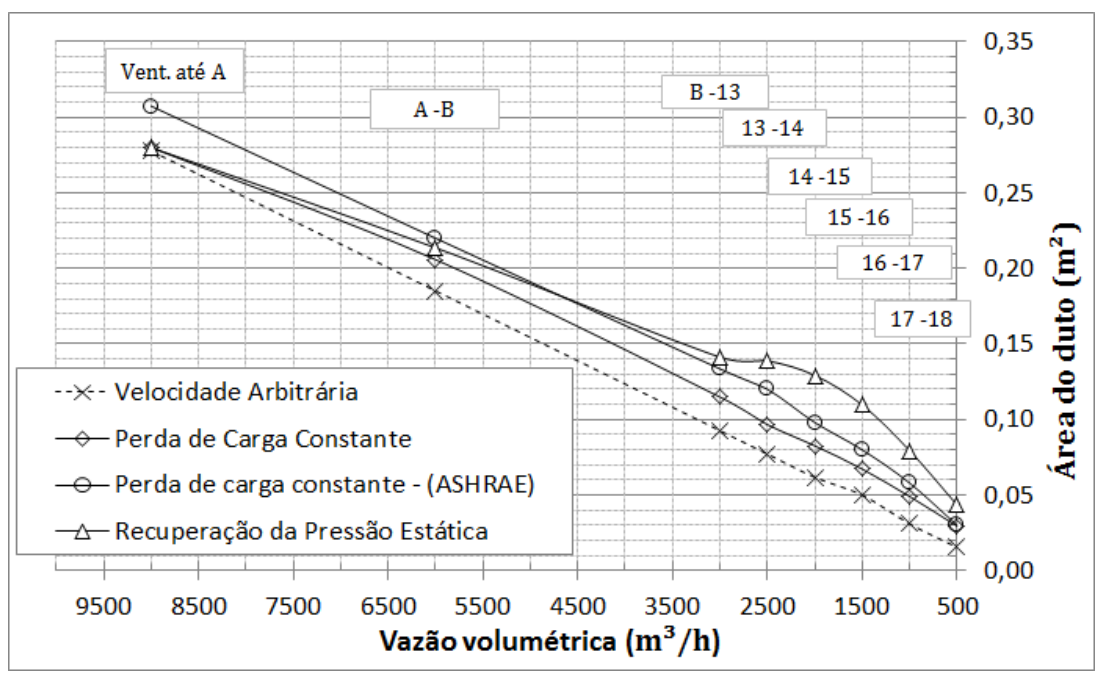

Figura 2 - Relação das áreas para o Método Aplicado.

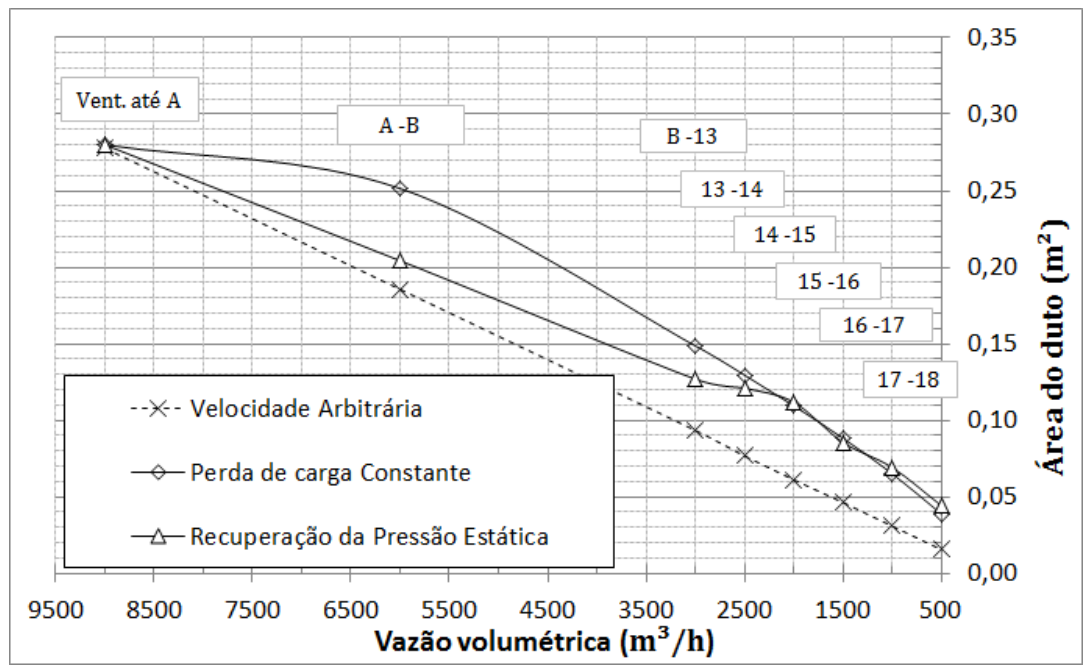

Figura 3 - Relação das áreas para o Método Direto.

A Figura (4) apresenta os resultados para o método de dimensionamento da velocidade arbitrária com as dimensões de cada trecho obtidas pelas duas formas de cálculo Método Aplicado e Direto. O resultado em termos das dimensões já era esperado, uma vez que nos dois procedimentos de cálculo o equacionamento é o mesmo. A diferença fica por conta da perda de carga, que normalmente superestima as perdas por atrito de acessórios (curvas, neste caso) em termos de comprimentos equivalentes. O valor da perda de carga para ambas as formas de cálculo é de 67,88 $\mathrm{mmH}_{2} \mathrm{O}$ pelo Método Aplicado, e 54,66 $\mathrm{mmH}_{2} \mathrm{O}$ pelo Método Direto, uma diferença de $24,19 \%$, enquanto os desvios nas dimensões não excederam $1 \%$. Embora seja instintivo considerar a perda de carga maior para o dimensionamento do sistema, é muito provável que se estará superestimando a queda de pressão do mesmo. Isto pode ser afirmado pelo fato de que é possível encontrar em diversas literaturas tabelas completamente diferentes no que diz respeito aos valores apresentados de queda de pressão para os mesmos componentes. Para fins de se ter uma estimativa da perda de carga de um sistema, quaisquer dos procedimentos podem ser adotados visto que a ordem de grandeza dos valores é a mesma. 


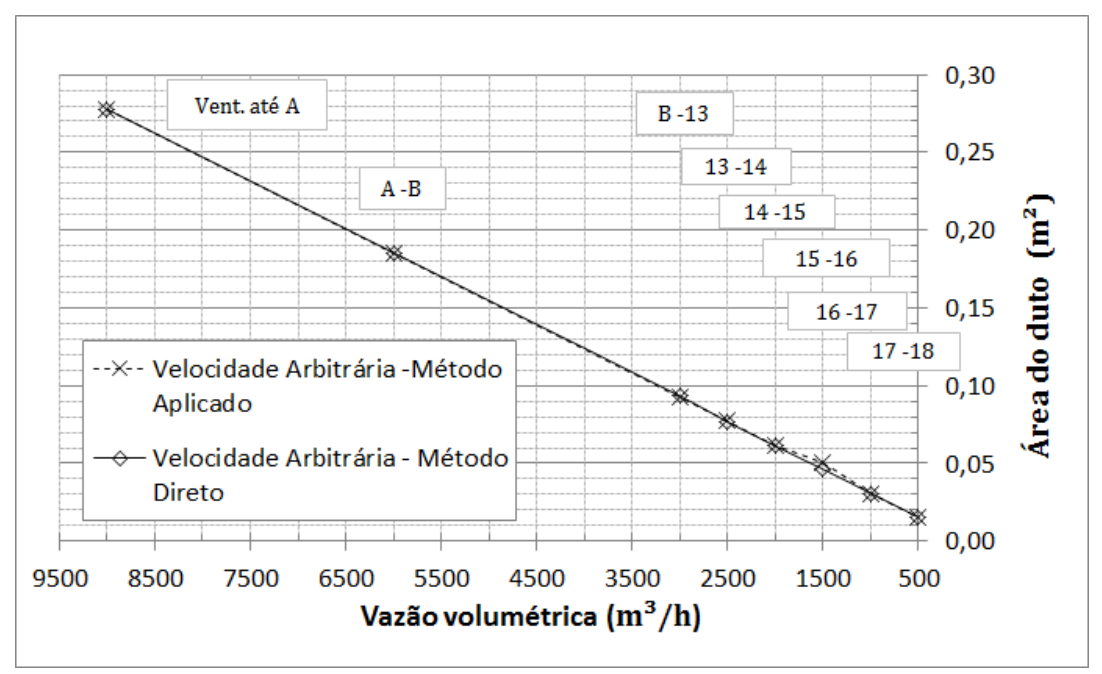

Figura 4 - Relação das áreas para o método da velocidade arbitrária.

As dimensões para as seções do sistema de dutos obtidas pelo método da Perda de Carga Constante são apresentadas na Figura (5). É possível observar, aqui, uma diferença notável entre os resultados para cada procedimento de cálculo. Este comportamento já era esperado visto que um dos métodos para procedimento de cálculo utiliza mais elementos gráficos e tabelas, gerando maior probabilidade de incertezas. Os procedimentos propostos pela ASHRAE (2013) [11], baseados em correlações aproximadas por dados ajustados experimentalmente, se aproximam um pouco mais da solução direta, no entanto também carregam incertezas provenientes dos ajustes e das tabelas de comprimentos equivalentes para acessórios.

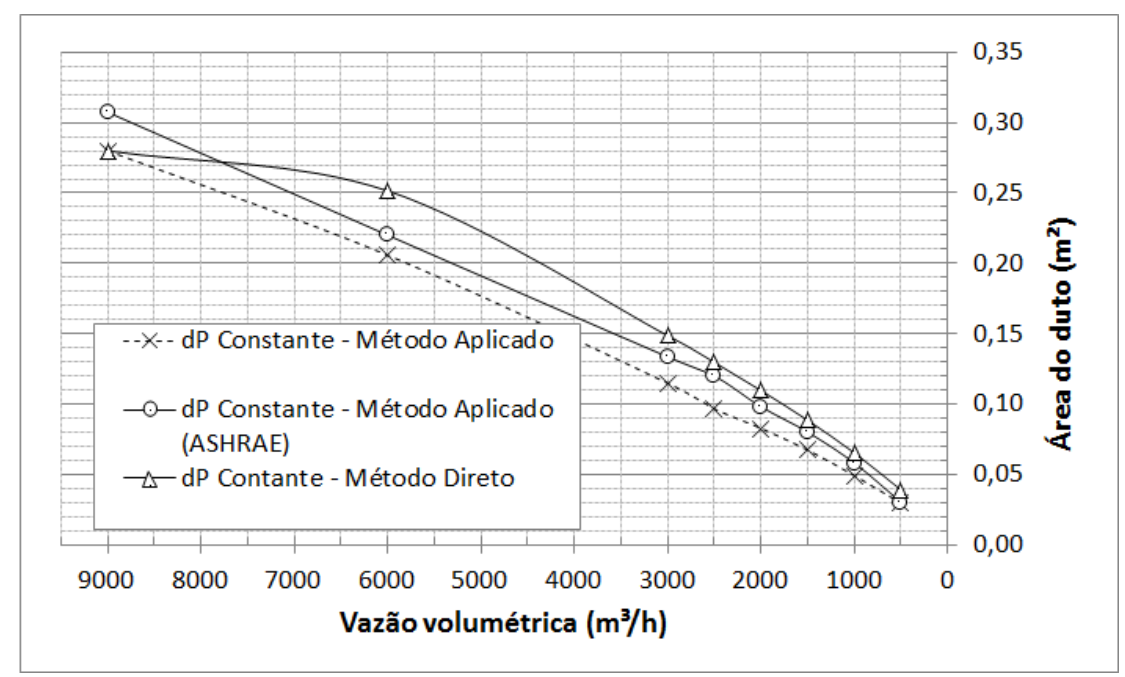

Figura 5 - Relação das áreas para o método da perda de carga constante.

A Figura (6) traz o comportamento dos desvios entre as seções dos trechos do sistema obtidas pelo dimensionamento com base em correlações da ASHRAE e aquele obtido a partir do Método Direto. Observa-se que os desvios em relação a solução clássica não apresentam uma tendência no comportamento, com uma variação que não é previsível. De toda forma, este comportamento é resultante, principalmente, pelas dimensões calculadas a partir da solução clássica, uma vez que os resultados obtidos através das correlações (ASHRAE) apresentam uma tendência linear. Os procedimentos de ajustes para obtenção das correlações fornecidas em ASHRAE (2013) [11] não são apresentados, bem como as formas de obtenção dos dados tabelados ou graficados. Não se pode afirmar que o dimensionamento está errado por uma ou outra solução, pois tratam-se de abordagens diferentes. A solução de forma direta é mais teórica, ou seja, acredita-se que descreve os fenômenos 
conforme definidos na mecânica dos fluidos, porém todas as questões contidas em experimentos reais não conseguem ser levadas em consideração. Os desvios observados ficam entre 7,4\% e $22,6 \%$, sendo o valor maior para a terminação do sistema de dutos. Em relação aos valores obtidos para as perdas de carga, a solução proposta pelas correlações fornece uma perda de carga de 8,06 $\mathrm{mmH}_{2} \mathrm{O}$, enquanto que o Método Direto conduziu a um valor de $9,93 \mathrm{mmH}_{2} \mathrm{O}$, um desvio de $23,2 \%$. A menor queda de pressão obtida pelas correlações, bem como as dimensões menores são explicadas pela maneira em como a metodologia é proposta. As correlações são divididas em sistemas de alta e baixa pressão. Desta forma, para cada um deles existe um limite máximo de velocidade do ar a ser respeitado, ou seja, a velocidade é uma variável resultante e não imposta. Em ambos os procedimentos de cálculo, a velocidade inicial é conhecida e determinada a partir de recomendações de norma. Estas diferenças de procedimento explicam os desvios maiores em relação às dimensões, bem como a influência deles na perda de carga. A variação das velocidades em cada trecho é apresentada na Figura (7).

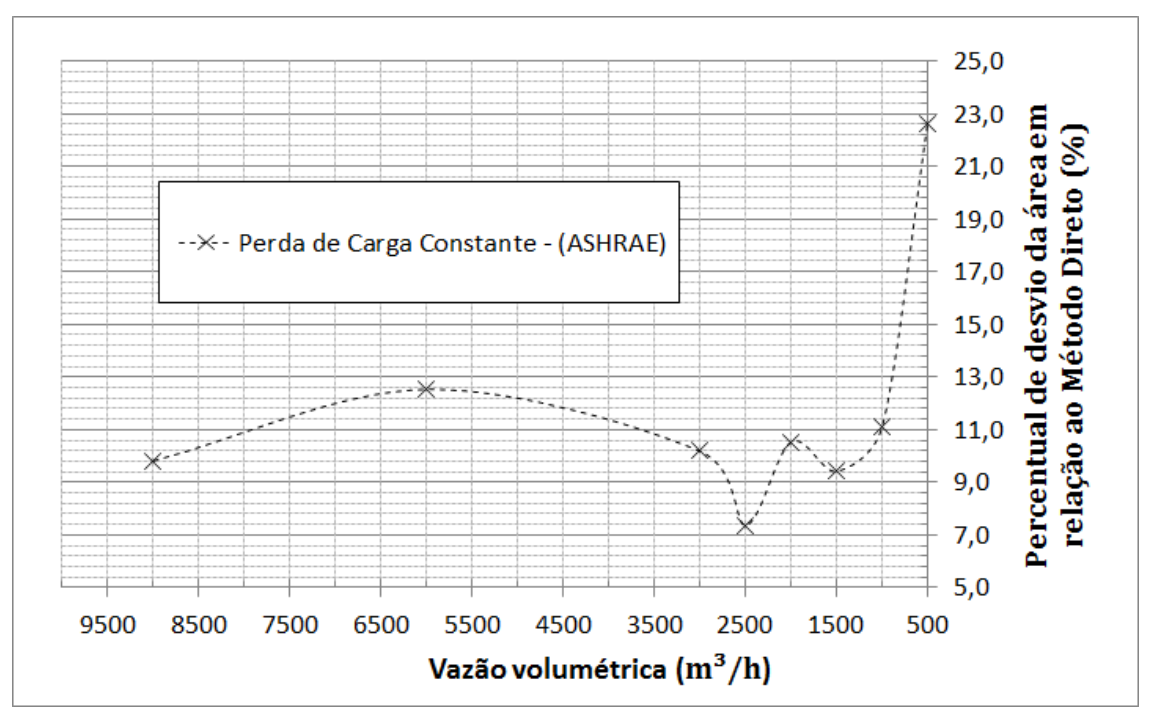

Figura 6-Desvio percentual das dimensões do sistema obtidos pelo método da perda de carga constante baseado em correlações da ASHRAE (2013) [11] em relação ao Método Direto.

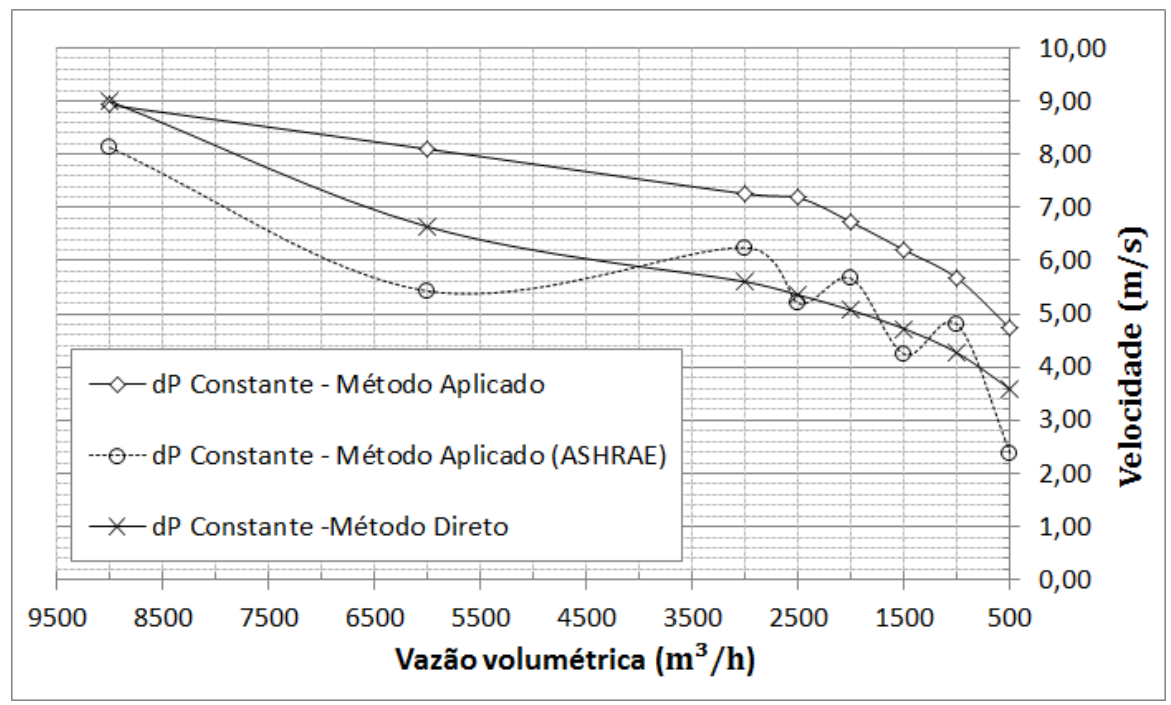

Figura 7 - Relação das velocidades para os métodos da perda de carga constante.

A mesma avaliação comparativa é feita entre o Método Aplicado, proposto em (Carrier, 1972) [13] e o Direto. A Figura (8) apresenta os desvios relativos às dimensões de cada trecho de duto para o primeiro. À medida que se aproxima do final do sistema de dutos os desvios aumentam 
significativamente em termos percentuais. Neste caso, embora o percentual na última saída seja de $24,3 \%$ é preciso ressaltar que do ponto de vista construtivo as diferenças dimensionais das áreas entre as duas formas de solução não é notória. O percentual, aparentemente, elevado se dá pelo fato de termos valores de áreas pequenos fisicamente, Figura (5). No entanto, é fato que as duas formas de dimensionamento geram sistemas diferentes. As perdas de cargas obtidas foram 10,58 $\mathrm{mmH}_{2} \mathrm{O}$ e $9,93 \mathrm{mmH}_{2} \mathrm{O}$ para as soluções Aplicada e Direta, respectivamente. O desvio destes valores está em $6,55 \%$, e pode ser considerado pouco significativo em termos aplicados.

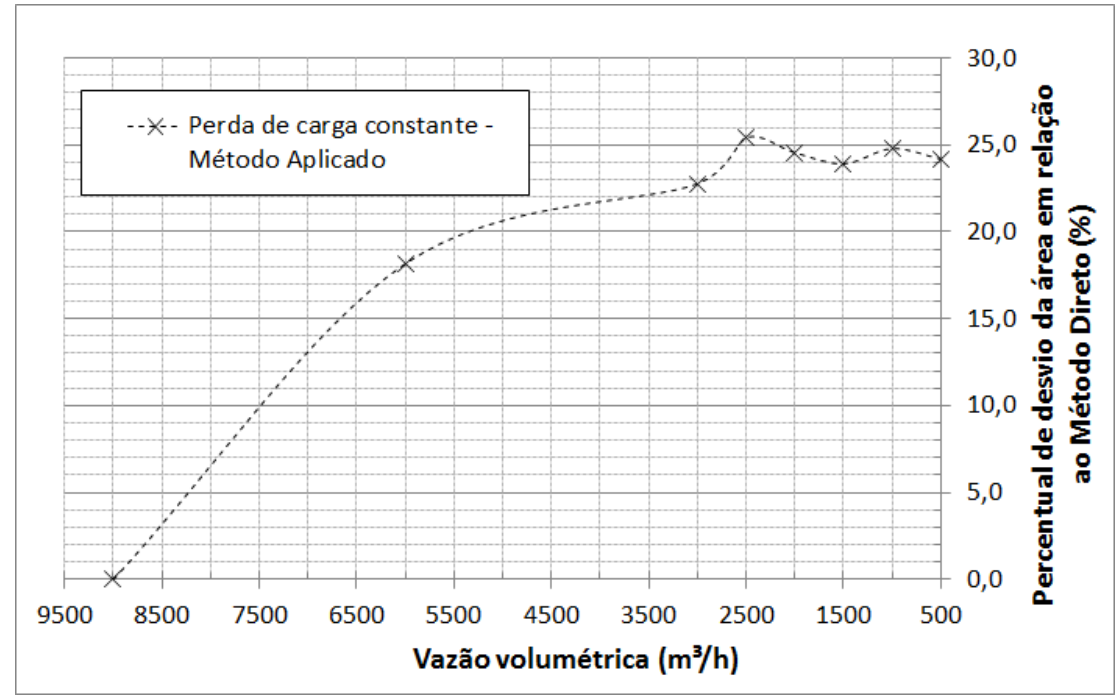

Figura 8 - Desvio percentual das dimensões do sistema obtidos pelo método da perda de carga constante pelo Método Aplicado em relação ao Método Direto.

A Figura (9) apresenta as variações das dimensões para o método da Recuperação da Pressão Estática obtidas pelas duas formas de solução já empregadas para os métodos anteriores. Para este terceiro método é, também, observada uma inversão resultados. Até então, os dimensionamentos efetuados a partir da metodologia Direta apresentaram maiores seções de duto em relação às dimensões encontradas pelo Método Aplicado, o que não é observado neste caso. Os maiores desvios aqui são observados logo no início da ramificação (saindo do duto principal - trechos 13 a 16), chegando a $28,16 \%$. Estes valores, aparentemente, significativos possuem uma explicação bastante provável. Dentre os três métodos, o da Recuperação da Pressão Estática, quando desenvolvido a partir de gráficos e tabelas, é o que mais se utiliza de fontes para consultas gráficas. Infelizmente não existem gráficos e tabelas com mesmos padrões de literatura para literatura, bem como pequenos desvios visuais nas leituras podem acarretar em grandes variações dimensionais finais. Ainda assim, mesmo com desvios percentuais relativamente elevados em determinado trecho do sistema, Figura (10), os valores absolutos das dimensões não são exorbitantemente diferentes, Figura (9).

Com relação às perdas de carga, a solução pelo Método Aplicado forneceu o valor de 3,78 $\mathrm{mmH}_{2} \mathrm{O}$, enquanto a pelo Método Direto de 3,45 $\mathrm{mmH}_{2} \mathrm{O}$, um desvio de 9,57\%.

A Tabela (1) apresenta de forma resumida os valores referentes às perdas de carga obtidas para todos os casos de dimensionamento do sistema hipotético deste trabalho. 


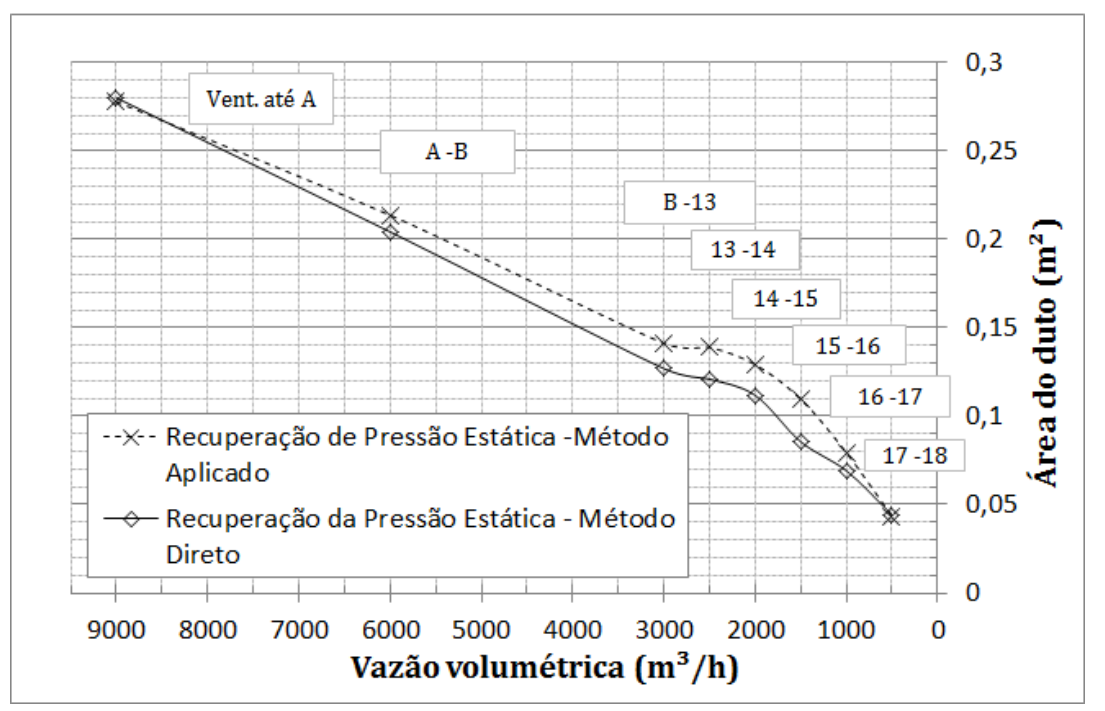

Figura 9 - Relação das áreas para o método da recuperação da pressão estática.

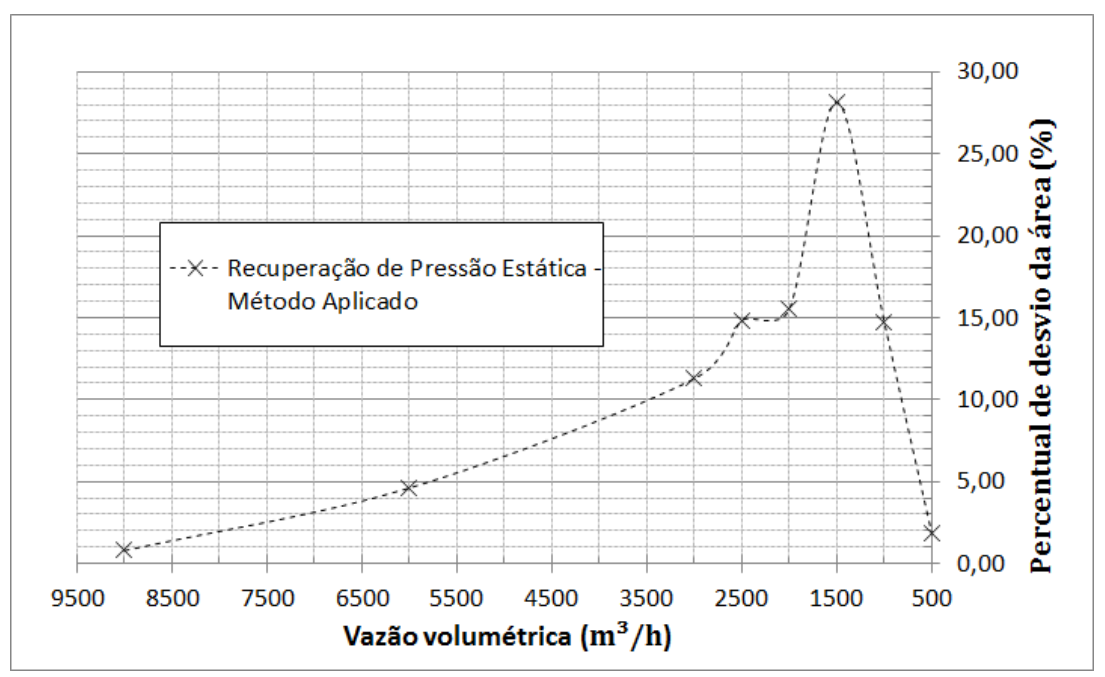

Figura 10 - Desvio percentual das dimensões do sistema obtidos pelo método da recuperação da pressão estática pelo Método Aplicado em relação ao Método Direto

Tabela 1 - Perda de carga total para cada método de ambas as técnicas de cálculo.

\begin{tabular}{ccc}
\hline \multicolumn{3}{c}{ Perda de Carga do Sistema (mmca) } \\
\hline Método & Método Aplicado & Método Direto \\
\hline Velocidade Arbitrária & 67,88 & 54,66 \\
\hline $\begin{array}{c}\text { Perda de Carga Constante } \\
\text { Recuperação da Pressão } \\
\text { Estática }\end{array}$ & $\begin{array}{c}8,06 \\
\text { (ASHRAE) }\end{array}$ & 9,93 \\
\hline
\end{tabular}




\section{CONCLUSÃO}

Este trabalho apresentou o dimensionamento de um sistema de distribuição de ar hipotético. Os métodos de dimensionamento avaliados foram: Velocidade Arbitrária, Perda de Carga Constante e Recuperação da Pressão Estática. Cada um dos métodos de dimensionamento foi avaliado perante duas formas de procedimento para solução: Método Aplicado, baseado fundamentalmente em tabelas e gráficos e; Método Direto, fundamentado na solução das equações advindas da mecânica dos fluídos em conjunto com algumas correlações empíricas. A segunda forma de solução foi implementada em linguagem de programação Phyton.

Os resultados foram analisados na forma comparativa das duas formas de solução expostas e avaliaram tanto as variações dimensionais quanto as perdas de carga totais do sistema.

Foram observados percentuais de desvios, aparentemente, significativos em relação às dimensões do sistema para as duas formas de solução. No entanto, em todos os casos, os valores absolutos dimensionais são toleráveis, visto que os maiores percentuais dizem respeito aos trechos em que as dimensões apresentam valores menores, dando ao caráter percentual uma magnitude virtualmente maior. Ainda com relação às dimensões são notadas tendências semelhantes para mesmos métodos quando comparados os procedimentos de solução Aplicada e Direta, o que pode indicar boa concordância, na descrição do comportamento do sistema, entre as duas formas de solução.

Os valores referentes às perdas de carga totais (teóricas) obtidas pelas soluções apontam para uma tendência de que o procedimento considerado aplicado superestima as quedas de pressão do sistema. Isto, em termos práticos, significaria a seleção de um equipamento com maior potência do que o necessário para manter o sistema em funcionamento, consequentemente maior custo de projeto e operacional. De certa forma, essa superestimativa das pressões de operação era esperada, uma vez que os procedimentos desta solução baseiam-se fundamentalmente em dados tabelados e graficados a partir de ajustes experimentais. A disponibilidade, em literatura, destes dados (tabelas, figuras, gráficos, etc.) é o principal ponto de possibilidade para variação dos resultados, pois as diferenças encontradas entre os materiais para consulta podem apresentar variações desde a escala de avaliação até a qualidade da impressão tipográfica destes.

Desta forma, conforme discutido anteriormente, embora os desvios percentuais dos valores de dimensionamento de um sistema de dutos tenham se apresentado relativamente elevados para algumas regiões, em termos absolutos os mesmos não devem ser significativos para um escoamento de ar. Assim, a seleção a respeito de qual procedimento utilizar para o dimensionamento deste tipo de sistema fica a critério do projetista, dando preferência para a maneira mais conveniente em termos de custo tanto operacionais quanto de tempo de projeto. Sem dúvida, para casos em que já se possuam códigos computacionais ou softwares para dimensionamento, a solução Aplicada com o auxílio computacional é significativamente mais dinâmica e, consequentemente, eficiente. No entanto, nem sempre tem-se equipamento disponível ou tempo para programação, enquanto que gráficos e tabelas são facilmente encontrados em buscas simples até mesmo na internet.

\section{REFERÊNCIAS BIBLIOGRÁFICAS}

1. Sugarman SC. Testing and Balancing Hvac Air and Water Systems, 4a Ed., Fairmont Press, Distributed by Taylor \& Francis, Lilburn, GA, Boca Raton, FL, 2006. 389p.

2. ASHRAE Handbook: Heating, Ventilating, and Air-conditioning Applications: American Society of Heating, Refrigerating and Air-conditioning Engineers, 2007. 1104p.

3. Enteria N, Mizutani K.. The role of the thermally activated desiccant cooling technologies in the issue of energy and environment. Renew Sustain Energy. 2011 May;15(4):2095-2122, doi:10.1016/j.rser.2011.01.013.

4. Qi R, Lu L, Yang H. Investigation on air-conditioning load profile and energy consumption of desiccant cooling system for commercial buildings in Hong Kong. Energy Build. 2012 Feb;49:509-518, doi:10.1016/j.enbuild.2012.02.051.

5. Grondzik WT. Air-conditioning System Design Manual, 2a Ed., ASHRAE Inc., Atlanta, USA; 2007. $416 \mathrm{p}$.

6. Sane HS, Haugstetter C, Bortoff SA, Building HVAC control systems - role of controls and optimization in: American Control Conference, 2006. Proceedings of the 2006 American Control Conference; 2006 
Jun. 14-16; Minneapolis, MN, IEEE Conference Publications; p. 1121-1126. doi: $10.1109 /$ acc. 2006.1656367

7. Knight I, Why energy monitoring and feedback is essential to achieve sustained energy efficiency in eu buildings, REHVA Eur. HVAC J., 2014 Jan; 01:16-19.

8. Woradechjumroen D, Yu YB, Ly HR, Yu DH, Yang HJ. Analysis of HVAC system oversizing in commercial buildings through field measurements. Energy Build. 2014 Feb;69:131-143, doi: 10.1016/j.enbuild.2013.10.015.

9. Wang SK.. Handbook of Air Conditioning and Refrigeration, 2a Ed., McGraw-Hill, Two Penn Plaza, New York, NY, USA, 2000. 1401p.

10. Bell AA. HVAC Equations, Data and Rules of Thumb, 2a Ed., McGraw-Hill, Two Penn Plaza, New York, NY, USA, 2007. 590p.

11. ASHRAE Handbook: Fundamentals: American Society of Heating, Refrigerating and Air-Conditioning Engineers, 2013. 1000p.

12. Costa EC. Ventilação. $1^{\mathrm{a} e d . ~ S a ̃ o ~ P a u l o: ~ E d g a r d ~ B l u c h e r, ~ 2005 . ~ 271 p . ~}$

13. Carrier Air Conditioning Company. Carrier System Design Manual Vol.1. New York, 1972, 368p.

14. Fox RW, McDonald AT, Pritchard PJ. Introdução à Mecânica dos Fluidos. $8^{\mathrm{a}}$ Ed., Rio de Janeiro: LTC, 2014. 871p.

15. Miller R, Miller MR. Ar-Condicionado e Refrigeração, $2^{\mathrm{a}}$ Ed., Rio Janeiro: LTC, 2014. 565p.

16. White FM. Mecânica dos Fluidos, 6ª Ed., Porto Alegre: AMGH, 2011.880p.

17. Churchill SW. Empirical expressions for the shear stress turbulent flow in commercial pipe. AIChE Journal. 1973 Mar;19(2):375-376, doi 10.1002/aic.690190228.

18. Churchill SW. Friction-factor equation spans all fluid flow regimes. Chem Eng. 1977 Nov;84(24):91-92.

19. Moody LF. Friction factors for pipe flow. Trans. ASME, 1944 Nov; 66(8):671-684.

20. Moddy LF. An approximate formula for pipe friction factors. Trans. ASME. 1947 Mar;69(12):10051011.

21. Colebrook CF., Turbulent flow in pipes with particular reference to the transition region between the smooth and rough pipe laws. J Inst Civil Eng. 1939 May;11(4):133-156.

22. Wood DJ. An explicit friction factor relationship. Civil. Engineering-ASCE, 1966 Dec; 36(12):60-61. 